\title{
EXTENSIONS OF BEST APPROXIMATION AND COINCIDENCE THEOREMS
}

\author{
SEHIE PARK \\ Department of Mathematics \\ Seoul National University \\ Seoul 151-742, Korea
}

(Received December 15, 1995)

ABSTRACT. Let $X$ be a Hausdorff compact space, $E$ a topological vector space on which $E^{*}$ separates points, $F: X \rightarrow 2^{E}$ an upper semicontinuous multifunction with compact acyclic values, and $g: X \rightarrow E$ a continuous function such that $g(X)$ is convex and $g^{-1}(y)$ is acyclic for each $y \in g(X)$. Then either (1) there exists an $x_{0} \in X$ such that $g x_{0} \in F x_{0}$; or (2) there exist an $\left(x_{0}, z_{0}\right)$ on the graph of $F$ and a continuous seminorm $p$ on $E$ such that

$$
0<p\left(g x_{0}-z_{0}\right) \leq p\left(y-z_{0}\right) \text { for all } y \in g(X) \text {. }
$$

A generalization of this result and its application to coincidence theorems are obtained. Our aim in this paper is to unify and improve almost fifty known theorems of others.

KEY WORDS AND PHRASES: multifunction, upper semicontinuous (u.s.c.), acyclic, convex space, admissible class, best approximation, metric projection, inward [outward] set.

1991 AMS SUBJECT CLASSIFICATION CODES: 41A65, 47H10, 54C60, 54H25, 55M20.

\section{INTRODUCTION}

One of the most interesting extensions of Ky Fan's best approximation theorems [1] was due to Prolla [2] for two functions. Subsequently, a number of its generalizations or variations followed, and some applications to coincidence theory were also given. See [3-8].

On the other hand, recently there have appeared some best approximation or fixed point theorems for maps whose domains and ranges have different topologies; for example, see [9-17]. Moreover, there have also appeared some generalizations of such results for two maps and two different space settings; for example $[3,18]$.

Usually, those results are obtained for single-valued maps or convex-valued upper semicontinuous multifunctions. However, more recently, the author $[11,13,19]$ showed that some of such best approximation and fixed point theorems can be extended for a large "admissible" class of multifunctions.

In the present paper, we obtain best approximation and coincidence theorems for such large class of multifunctions and two different space settings. Our new results are general enough to subsume more than fifty known results of other authors as particular cases. 


\section{PRELIMINARIES}

A multifunction or set-valued map (simply, map) $F: X \rightarrow 2^{Y}$ is a function with nonempty set-values $F x \subset Y$ for each $x \in X$. The set $\{(x, y): y \in F x\}$ is called either the graph of $F$ or, simply, $F$. So $(x, y) \in F$ if and only if $y \in F x$.

For topological spaces $X$ and $Y$, a map $F: X \rightarrow 2^{Y}$ is upper semecontinuous (u.s.c.) if, for each closed set $B \subset Y, F^{-1}(B)=\{x \in X: F x \cap B \neq \emptyset\}$ is closed in $X$. It is well-known that if $Y$ is compact Hausdorff and $F x$ is closed for each $x \in X$, then $F$ is u.s.c. if and only if the graph of $F$ is closed in $X \times Y$. A nonempty topological space is acyclic if all of its reduced Cech homology groups over rationals vanish.

A convex space $C$ is a nonempty convex set with any topology that induces the Euclidean topology on the convex hulls of its finite subsets. Such convex hulls are called polytopes.

Given a class $\mathbb{X}$ of maps, $\mathbb{X}(X, Y)$ denotes the set of all maps $F: X \rightarrow 2^{Y}$ belonging to $\mathbb{X}$, and $\mathbb{X}_{c}$ the set of all finite composites of maps in $\mathbb{X}$.

A class $\mathfrak{A}$ of maps is one satisfying the following:

(i) $\mathfrak{A}$ contains the class $\mathbb{C}$ of (single-valued) continuous functions:

(ii) each $F \in \mathfrak{A}_{c}$ is u.s.c. and compact-valued; and

(iii) for any polytope $P$, each $f \in \mathfrak{A}_{c}(P, P)$ has a fixed point.

Examples of $\mathfrak{A}$ are $\mathbb{C}$, the Kakutani maps $\mathbb{K}$ (with convex values in convex spaces), the acyclic maps $\mathbb{V}$ (with acyclic values), the approachable maps $\mathbb{A}$ in topological vector spaces [20-22], admissible maps in the sense of Górniewicz [23], permissible maps in Dzedzej [24], and others. Moreover, we define

$F \in \mathfrak{A}_{c}^{\sigma}(X, Y) \Longleftrightarrow$ for any $\sigma$-compact subset $K$ of $X$, there is a $\Gamma \in \mathfrak{A}_{c}(K, Y)$ such that $\Gamma x \subset F x$ for each $x \in K$.

$F \in \mathfrak{A}_{c}^{\kappa}(X, Y) \Longleftrightarrow$ for any compact subset $K$ of $X$, there is a $\Gamma \in \mathfrak{A}_{c}(K, Y)$ such that $\Gamma x \subset F x$ for each $x \in K$.

A class $\mathfrak{A}_{c}^{\kappa}$ is said to be admissible. Note that $\mathfrak{A} \subset \mathfrak{A}_{c} \subset \mathfrak{A}_{c}^{\sigma} \subset \mathfrak{A}_{c}^{\kappa}$. Examples of $\mathfrak{A}_{c}^{\sigma}$ are $\mathbb{K}_{c}^{\sigma}$ due to Lassonde [25] and $\mathbb{V}_{c}^{\sigma}$ due to Park et al. [26]. Note that $\mathbb{K}_{c}^{\sigma}$ includes classes $\mathbb{K}, \mathbb{R}$, and $\mathbb{T}$ in [25]. The approximable maps recently due to Ben-El-Mechaiekh and Idzik [27] belong to $\mathfrak{A}_{c}^{\kappa}$. Therefore, any compact-valued u.s.c. map $F: X \rightarrow 2^{E}$, where $E$ is a locally convex t.v.s. and $X \subset E$, belongs to $\boldsymbol{A}_{c}^{\kappa}$ if its values are all convex, contractible, decomposable, or $\infty$-proximally connected. See [27].

Let $E=(E, \tau)$ be a topological vector space, $E^{*}$ its topological dual, and $S(E)=S(E, \tau)$ the family of all continuous seminorms on $(E, \tau)$. Let $w$ denote the weak topology of $E$. We say that $E^{*}$ separates points of $E$ if for each $x \in E$ with $x \neq 0$, there exists a $\phi \in E^{*}$ such that $\phi(x) \neq 0$; that is, if $x \neq 0$, then $p(x)>0$ for some $p \in S(E, w) \subset S(E, \tau)$ by taking $p(x)=|\phi(x)|$ for all $x \in E$.

The following is due to the author $[19, \mathrm{II}]$ :

THEOREM 2.1. Let $X$ be a compact convex subset of a topological vector space $E$ on which $E^{*}$ separates points. Then any $F \in \mathfrak{A}_{c}^{\kappa}(X, X)$ has a fixed point.

Let $C$ be a nonempty subset of a Hausdorff topological vector space $E$ and $p \in S(E)$. For each $y \in E$, define $d_{p}(y, C)=\inf \{p(y-x): x \in C\}$ and the set of best approximations to $y \in E$ 
from $C$ by $Q_{p}(y)=\left\{x \in C: p(y-x)=d_{p}(y, C)\right\}$. The multifunction $Q_{p}$ thus defined is called the metric projection onto $C$ if $Q_{p}(y) \neq \emptyset$ for each $y \in E$. It is well-known that if $C$ is compact convex, then the metric projection $Q_{p}: E \rightarrow 2^{C}$ belongs to $\mathbb{K}(E, C)$.

In $(E, \tau)$, let $\mathrm{Bd}$, Int, and - denote the boundary, interior, and closure, respectively, with respect to $\tau$.

The inward and outward sets of $X \subset E$ at $x \in E, I_{X}(x)$ and $O_{X}(x)$, are defined as follows:

$$
\begin{aligned}
I_{X}(x) & =\{x+r(u-x): u \in X, r>0\} \\
O_{X}(x) & =\{x+r(u-x): u \in X, r<0\} .
\end{aligned}
$$

For a topological space $X$, a real function $f: X \rightarrow \mathbb{R}$ is lower semicontinuous (l.s.c.) if $\{x \in X: f x>r\}$ is open for each $r \in \mathbb{R}$.

The following is well known:

LEMMA 2.2. Let $X$ and $Y$ be topological spaces, $h: X \times Y \rightarrow \mathbb{R}$ l.s.c. and $F: X \rightarrow 2^{Y}$ a compact-valued u.s.c. multifunction. Then $x \mapsto \inf \{h(x, y): y \in F x\}$ is l.s.c. on $X$.

\section{MAIN RESULTS}

From Theorem 2.1, we obtain the following generalization of many best approximation and fixed point theorems:

THEOREM 3.1. Let $X$ be a Hausdorff compact convex space, $E=(E, \tau)$ a topological vector space on which $E^{*}$ separates points, $F \in \mathfrak{A}_{c}^{\kappa}(X,(E, w))$, and $g \in \mathbb{C}(X,(E, w))$ such that $g(X)$ is convex. Suppose that either

(I) $g^{-1}(y)$ is convex for $y \in g(X)$ and $\mathbb{K}(g(X), X) \subset \mathfrak{A}(g(X), X)$; or

(II) $g^{-1}(y)$ is acyclic for $y \in g(X)$ and $\mathbb{V}(g(X), X) \subset \mathfrak{A}(g(X), X)$.

Then either

(1) there exists an $x_{0} \in X$ such that $g x_{0} \in F x_{0}$; or

(2) there exist an $\left(x_{0}, z_{0}\right) \in F$ and a $p \in S(E, w)$ such that $g x_{0} \in \operatorname{Bd} g(X)$ and

$$
0<p\left(g x_{0}-z_{0}\right) \leq p\left(y-z_{0}\right) \text { for all } y \in \bar{I}_{g(X)}\left(g x_{0}\right) \text {. }
$$

PROOF. Since $X$ is compact, we may assume that $F \in \mathfrak{A}_{c}(X,(E, w))$. Since the graph of $g^{-1}: g(X) \rightarrow 2^{X}$ is closed in $g(X) \times X$ and $X$ is compact Hausdorff, we know that $g^{-1}$ is u.s.c. and $g^{-1}(y)$ is closed for each $y \in g(X)$. Therefore, either (I) $g^{-1} \in \mathbb{K}(g(X), X)$ if $g^{-1}$ is convex-valued; or (II) $g^{-1} \in \mathbb{V}(g(X), X)$ if $g^{-1}$ is acyclic-valued. Let $p \in S(E, w)$. Consider the composite of multifunctions

$$
g(X) \stackrel{g^{-1}}{\longrightarrow} X \stackrel{F}{\rightarrow}(E, w) \stackrel{Q_{p}}{\longrightarrow} g(X) .
$$

Since $g(X)$ is a weakly compact convex subset of a Hausdorff topological vector space $(E, w)$, the metric projection $Q_{p}$ belongs to $\mathbb{K}((E, w), g(X))$. Hence, in any case we have

$$
Q_{p} F g^{-1} \in \mathfrak{A}_{c}^{\kappa}(g(X), g(X)),
$$


which has a fixed point $y_{0} \in g(X)$ by Theorem 2.1. Then $y_{0} \in\left(Q_{p} F\right) x_{0}$ for some $x_{0} \in g^{-1}\left(y_{0}\right)$; and hence $g x_{0} \in Q_{p} z_{0}$ for some $z_{0} \in F x_{0}$, which is equivalent to

$$
p\left(g x_{0}-z_{0}\right) \leq p\left(y-z_{0}\right) \text { for all } y \in g(X) .
$$

This inequality holds for all $y \in \bar{I}_{g(X)}\left(g x_{0}\right)$ by the method in $[6,7,11-13,19,28,29]$. If $g x_{0} \in$ Int $g(X)$, then $\bar{I}_{g(X)}\left(g x_{0}\right)=E$. Therefore, by putting $y=z_{0}$ in the above inequality, we have $p\left(g x_{0}-z_{0}\right)=0$.

Suppose that (2) does not hold. Then for each $p \in S(E, w)$, there exists an $(x, z) \in F$ such that $p(g x-z)=0$; that is,

$$
F[p]=\left\{x \in X: d_{p}(g x, F x)=0\right\} \neq \emptyset .
$$

Considering $(g(X), E)$ instead of $(X, Y)$ in Lemma 2.2, put

$$
h(g x, z)=p(g x-z) \text { for } \quad x \in X, z \in E .
$$

Then $x \mapsto d_{p}(g x, F x)$ is l.s.c. Therefore, $F[p]$ is closed in $X$. Moreover, for a finite subset $\left\{p_{1}, p_{2}, \ldots, p_{n}\right\}$ of $S(E, w)$, we have $p=\sum_{t=1}^{n} p_{1} \in S(E, w)$ and $F[p]=F\left[\sum_{t=1}^{n} p_{\mathrm{t}}\right] \subset \bigcap_{t=1}^{n} F\left[p_{1}\right]$. Therefore, $\{F[p]: p \in S(E, w)\}$ is a family of closed subsets of $X$ with the finite intersection property. Since $X$ is compact, there exists a $u \in \bigcap\{F[p]: p \in S(E, w)\}$. Now we claim that $g u \in F u$.

Suppose that $g u \notin F u$. Then the origin 0 does not belong to the compact set $K=g u-F u$ of $(E, w)$. Let $z \in K$. Since $E^{*}$ separates points of $(E, w)$, there exists a $\phi \in E^{*}=(E, w)^{*}$ such that $\phi(z) \neq 0$. By putting $p_{z}(x)=|\phi(x)|$ for $x \in E$, we know that $p_{z} \in S(E, w)$ and $p_{z}(z)>0$. Since $p_{z}$ is continuous on $K$, there exists an open neighborhood $U_{z}$ of $z$ in $K$ such that $p_{z}(y)>0$ for every $y \in U_{z}$. Let $\left\{U_{z_{1}}, \ldots, U_{z_{k}}\right\}$ be a finite subcover of the cover $\left\{U_{z}\right\}_{z \in K}$ of $K$ and let $p_{u}=$ $\sum_{t=1}^{k} p_{z_{\imath}} \in S(E, w)$. Since $\left.p_{u}\right|_{K}$ is continuous, it attains its infimum on $K$. Since the infimum can not be zero, we have $d_{p_{u}}(g u, F u)>0$. This contradicts $u \in \bigcap\{F[p]: p \in S(E, w)\} \neq \emptyset$. This completes our proof.

REMARKS 3.1. 1. In Theorem 3.1, $F \in \mathfrak{A}_{c}^{\kappa}(X,(E, w))$ and $g: X \rightarrow(E, w)$ can be replaced by $F \in \mathfrak{A}_{c}^{\kappa}(X,(E, \tau))$ and $g: X \rightarrow(E, \tau)$, respectively, without affecting the conclusion.

2. If $F^{\prime} \in \mathfrak{A}_{c}^{\kappa}(X,(E, w))$, where $F^{\prime}$ is defined by $F^{\prime} x=2 g x-F x$ for $x \in X$, then the inward set in the conclusion (2) of Theorem 3.1 can be replaced by the corresponding outward set.

3. In Theorem 3.1, we assumed that $g: X \rightarrow(E, w)$ satisfies

(i) $g(X)$ is convex and $g^{-1}(y)$ is convex [or acyclic] for $y \in g(X)$. [18].

Particular forms of (i) were appeared in the literature as follows:

(ii) $g^{-1}([y, z])$ is convex for any $y, z \in g(X)$, where $[y, z]=\{\lambda y+(1-\lambda) z: 0 \leq \lambda \leq 1\}$. [18]

(iii) $g$ is affine (that is, $g\left(\lambda x_{1}+(1-\lambda) x_{2}\right)=\lambda g x_{1}+(1-\lambda) g x_{2}$ with $\left.0 \leq \lambda \leq 1\right)$. [3]

(iv) $g^{-1}(C)$ is convex (or empty) for any closed convex subset $C$ of $E$. [30]

Note that (iv) $\Longrightarrow$ (ii) and (iii) $\Longrightarrow$ (ii). Now, we show that (ii) $\Longrightarrow$ (i):

In fact, for any $y=[y, y] \in g(X), g^{-1}(y)=g^{-1}([y, y])$ is convex by (ii). We claim that $g(X)$ is convex. For any $g x_{1}, g x_{2} \in g(X)$, by (ii), $g^{-1}\left(\left[g x_{1}, g x_{2}\right]\right)$ is convex and contains $x_{1}$ and $x_{2}$. Therefore, $\left[x_{1}, x_{2}\right] \subset g^{-1}\left(\left[g x_{1}, g x_{2}\right]\right)$ or $g\left(\left[x_{1}, x_{2}\right]\right) \subset\left[g x_{1}, g x_{2}\right]$. This implies $\left[g x_{1}, g x_{2}\right]=$ $g\left(\left[x_{1}, x_{2}\right]\right) \subset g(X)$ since $X$ is convex and $g$ is continuous on the closed interval $\left[x_{1}, x_{2}\right]$. 
Consider two more conditions on $g: X \rightarrow(E, w)$ with respect to $p \in S(E, w)$ as follows :

(v) $g$ is almost p-quasiconvex if

$$
p(g(r x+(1-r) y)-u) \leq \max \{p(g x-u), p(g y-u)\}
$$

(vi) $g$ is almost p-affine if

$$
p(g(r x+(1-r) y)-u) \leq r p(g x-u)+(1-r) p(g y-u)
$$

for $x, y \in X, u \in E$, and $r \in(0,1)$.

Those concepts were appeared in $[2,4,14,31]$. Note that (iii) $\Longrightarrow(\mathrm{vi}) \Longrightarrow(\mathrm{v})$, and if $p$ is a norm and if $g(X)$ is convex, then $(\mathrm{v}) \Longrightarrow(\mathrm{i})$.

PARTICULAR FORMS 3.1. Theorem 1 generalizes, unifies, and improves many of wellknown best approximation and fixed point theorems. We list some major particular forms in the chronological order.

1. Fan [1, Theorem 1]: Let $E$ be locally convex, $X$ a nonempty compact convex subset of $E$, $F=f=\mathbb{C}(X, E)$, and $g=1_{X}$ the identity map.

2. Fan [1, Theorem 2]: $E$ is a normed vector space in the above.

3. Halpern [32, Theorem 20]: $X$ is a subset of a Banach space $E, g=1_{X}$, and $F \in \mathbb{V}(X, E)$ under some restrictions.

4. Kapoor [10, Theorem 2]: $X$ is a nonempty weakly compact convex subset of a normed vector space $E, F=f \in \mathbb{C}((X, w),(E,\|\cdot\|))$ [that is, strongly continuous], and $g=1_{X}$.

5. Fitzpatrick and Petryshyn [33, Theorem $3(\mathrm{i})]$ : $X$ is a subset of a strictly convex Banach space $E, g=1_{X}$, and $F \in \mathbb{V}(X, E)$.

6. Reich [34, Theorems 1 and 2]: $X$ is a subset of a locally convex Hausdorff topological vector space, $g=1_{X}$, and $F \in \mathbb{K}(X, E)$.

7. Prolla [2, Theorem]: $X$ is a subset of a normed vector space $E, F=f \in \mathbb{C}(X, E)$, and $g \in \mathbb{C}(X, X)$ is an almost affine surjection.

8. Sehgal and Singh [15, Theorem 1 and Corollary 1], Sehgal et al. [16, Corollary 1]: $X$ is a nonempty weakly compact convex subset of a real locally convex Hausdorff topological vector space $(E, \tau), F=f \in \mathbb{C}((X, w),(E, \tau))$ [that is, strongly continuous], and $g=1_{X}$.

9. Ha [18, Theorem 3]: $X$ is a compact convex subset of a Hausdorff topological vector space, $E$ a locally convex Hausdorff topological vector space, and $F=f, g \in \mathbb{C}(X, E)$ such that $g(X)$ and $g^{-1}(y)$ are convex for $y \in g(X)$.

10. Ha [35, Theorem 3]: $X$ is a nonempty compact convex subset of a locally convex Hausdorff topological vector space $E, g=1_{X}$, and $F \in \mathbb{K}(X, E)$.

11. Park [6, Theorem 2.1]: $X$ is a subset of a normed vector space $E$, and $F=f, g \in \mathbb{C}(X, E)$ such that $g(X)=X$ and $g$ is almost affine.

12. Lin [3, Theorem 1]: $X$ is a nonempty weakly compact convex subset of a locally convex Hausdorff topological vector space, $(E, \tau)$ is locally convex, $F=f \in \mathbb{C}(X,(E, \tau))$ [that is, strongly continuous], and $g \in \mathbb{C}(X,(E, w))$ [that is, weakly continuous] satisfies (ii) instead of (i).

13. Carbone [4, Theorem 1]: $X$ is a subset of a normed vector space $E, g \in \mathbb{C}(X, X)$ is an almost quasiconvex surjection, and $f \in \mathbb{C}(X, E)$. 
14. Carbone and Conti [5, Corollary 1]: $X$ is a subset of a Banach space $E, f \in \mathbb{C}(X, E)$. $g \in \mathbb{C}(X, X)$ is surjective, and $g^{-1}(y)$ is acyclic for each $y \in X$.

15. Sessa and Singh [8, Theorem 4]: $X$ is a subset of a normed linear space $E, f \in \mathbb{C}(X, E)$. and $g \in \mathbb{C}(X, E)$ such that $g(X)$ and $g^{-1}(y)$ are convex for $y \in y(X)$.

16. Sessa and Singh [8, Corollary 1]: $X$ is a nonempty weakly compact convex subset of a normed linear space $(E,\|\cdot\|), F=f \in \mathbb{C}((X, w),(E,\|\cdot\|))$, and $g \in \mathbb{C}((X, w),(E, w))$ such that $g^{-1}([y, z])$ is convex for any $y, z \in g(X)$.

17. Sessa and Singh [8, Corollary 2]: $X$ is a subset of a normed linear space $E$, and $F=$ $f, g \in \mathbb{C}(X, E)$ such that $g^{-1}([y, z])$ is convex for any $y, z \in g(X)$.

18. Ding and Tan [9, Theorem 4]: $X$ is a weakly compact convex subset of a locally convex Hausdorff topological vector space $(E, \tau), g=1_{X}$, and $F \in \mathbb{K}((X, w),(E, \tau))$.

19. Park [29, Theorem 3]: $X$ is a subset of $E, g=1_{X}$, and $F \in \mathbb{V}(X, E)$.

20. Park [19, II, Theorem 4]: $X$ is a subset of $E, g=1_{X}$, and $F \in \mathfrak{A}_{c}^{\kappa}(X, E)$.

21. Park [13, Theorems 2(I) and 3]: $X$ is a subset of $(E, \tau)$ for (II).

22. Ding and Tarafdar [36, Theorems 3.3 and $\left.3.3^{\prime}\right]: F \in \mathbb{K}(X, E)$.

From Theorem 3.1, we obtain the following coincidence theorem:

THEOREM 3.2. Let $X$ be a Hausdorff compact convex space, $E=(E, \tau)$ a topological vector space on which $E^{*}$ separates points, $F \in \mathfrak{A}_{c}^{\kappa}(X,(E, w))$, and $g \in \mathbb{C}(X,(E, w))$ such that $g(X)$ is convex. Suppose that either (I) or (II) of Theorem 3.1 holds. Then there exists an $x_{0} \in X$ such that $g x_{0} \in F x_{0}$ whenever one of the following conditions holds:

For each $x \in X$ with $g x \in \operatorname{Bd} g(X)$,

(0) for each $z \in F x$ and $p \in S(E, w), p(g x-z)>0$ implies

$$
p(g x-z)>d_{p}\left(z, \bar{I}_{g(X)}(g x)\right) .
$$

(i) for each $z \in F x$, there exists a number $\lambda$ (real or complex, depending on whether the vector space $E$ is real or complex) such that

$$
|\lambda|<1 \text { and } \lambda g x+(1-\lambda) z \in \bar{I}_{g(X)}(g x) .
$$

(ii) $F x \subset \bar{I}_{g(x)}(g x)$.

(iii) for each $z \in F x$, there exists a number $\lambda$ (as in (i)) such that

$$
|\lambda|<1 \text { and } \lambda g x+(1-\lambda) z \in g(X) .
$$

(iv) $F x \subset I F_{g(X)}(g x)=\{g x+c(u-g x): u \in g(X), \operatorname{Re}(c)>1 / 2\}$.

(v) $F x \subset g(X)$.

(vi) $F(X) \subset g(X)$.

PROOF. (0) Clear from Theorem 3.1.

(i) For each $p \in S(E, w)$ satisfying $p(g x-z)>0$, put $y:=\lambda g x+(1-\lambda) z \in \bar{I}_{g(x)}(g x)$. Then, we have

$$
d_{p}\left(z, \bar{I}_{g(X)}(g x)\right) \leq p(z-y)=|\lambda| p(g x-z)<p(g x-z) .
$$

Therefore (0) holds.

(ii) If $F x \subset \bar{I}_{g(x)}(g x)$, then for each $z \in F x$, we can choose $\lambda=0$ in (i). 
(iii) Since $g(X) \subset I_{g(X)}(g x)$, we clearly have (iii) $\Longrightarrow$ (i).

(iv) It is well known that (iv) $\Longleftrightarrow$ (iii) [37].

(v) If $F x \subset g(X)$, then for each $z \in F x$, we can choose $\lambda=0$ in (iii).

(vi) Clearly. we have (vi) $\Longrightarrow(v)$.

REMARKS 3.2. 1. If $F^{\prime} \in \mathfrak{A}_{c}^{\kappa}(X,(E, w))$, where $F^{\prime}$ is defined by $F^{\prime} x=2 g x-F x$ for $x \in X$, then the inward set in Theorem 3.2 can be replaced by the corresponding outward set.

2. If $g=1_{x}$ and $\mathfrak{A}_{c}^{\kappa}$ is replaced by $\mathbb{K}$ in Theorem $3.2(\mathrm{I})$, then the boundary conditions in Theorem 3.2 can be replaced by more general ones. See [19].

PARTICULAR FORMS 3.2. We list major particular forms of Theorem 3.2.

1. If $X$ is a subset of $(E, \tau), F \in \mathbb{K}((X, \tau),(X, \tau))$, and $g=1_{X}$, then the boundary condition (vi) holds trivially. In this case, Theorems 2.1 and 3.2 include historically well-known fixed point theorems of Brouwer (1912), Schauder (1927, 1930), Tychonoff (1935), Kakutani (1941), Bohnenblust and Karlin (1950), Fan (1952, 1964), Glicksberg (1952), Granas and Liu (1986), and many others. See [19].

2. Knaster et al. [38]: If $X=C^{n}$ is an $n$-cell in $E=\mathbb{R}^{n}, g=1_{X}$, and $f \in \mathbb{C}\left(C^{n}, \mathbf{R}^{n}\right)$ satisfies $f\left(\operatorname{Bd} C^{n}\right) \subset C^{n}$, then $f$ has a fixed point in $C^{n}$.

3. Eilenberg and Montgomery [39, Theorem 6]: If $X=C^{n}, E=\mathbf{R}^{n}, g=1_{X}$, and $F \in$ $\mathbb{V}\left(C^{n}, \mathbf{R}^{n}\right)$ satisfies $F\left(\operatorname{Bd} C^{n}\right) \subset C^{n}$, then $F$ has a fixed point in $C^{n}$.

4. Halpern [40] and Browder [41, Theorem 1]: $X$ is a subset of a locally convex Hausdorff topological vector space $E, g=1_{X}$, and $F=f \in \mathbb{C}(X, E)$ such that $f x \in I_{X}(x)$ [or $f x \in O_{X}(x)$ ] for $x \in X$.

5. Halpern and Bergman [42, Theorems 4.1 and 4.3]: $X$ is a subset of $E, g=1_{X}$, and $F=f \in \mathbb{C}(X, E)$ such that $f x \in \bar{I}_{X}(x)$ [or $f x \in \bar{O}_{X}(x)$ ] for $x \in X$.

6. Fan [1, Theorem 3]: $X$ is a subset of $E, E$ is locally convex, $F=f \in \mathbb{C}(X, E)$, and $g=1_{X}$ such that the boundary condition (iii) holds.

7. Halpern [32, Corollaries 21 and 22]: $X$ is a subset of a Banach space $E, g=1_{X}$. and $F \in \mathbb{V}(X, E)$ with $F x \subset \bar{I}_{X}(x)$ [or $F x \subset \bar{O}_{X}(x)$ ] for all $x \in X$.

8. Reich [37, Theorem 1.7], [43, Theorem 3.1]: $X$ is a subset of a locally convex Hausdorff topological vector space $E, g=1_{X}$, and $F \in \mathbb{K}(X, E)$ such that $F x \subset I F_{X}(x)=\{x+c(y-x)$ : $y \in X$ and $\operatorname{Re}(c)>1 / 2\}$.

9. Fitzpatrick and Petryshyn [33, Corollary 1]: $X$ is a subset of a strictly convex Banach space $E, g=1_{X}$, and $F \in \mathbb{V}(X, E)$ satisfies $F x \subset \bar{I}_{X}(x)$ for all $x \in X$.

10. Browder [44, Corollaries 1 and 2]: $X$ is a subset of a Banach space $E, g=1_{X}$, and $F=f \in \mathbb{C}(X, E)$ such that for each $x \in x$ with $x \neq f x$, there exists a $y \in I_{X}(x)$ satisfying $\|y-f x\|<\|x-f x\|$.

11. Sehgal and Singh [15, Corollary 2]: $X$ is a weakly compact convex subset of a locally convex Hausdorff topological vector space $(E, \tau), g=1_{X}$, and $F=f \in \mathbb{C}((X, w),(E, \tau))$ [that is, strongly continuous] with $f(\operatorname{Bd} X) \subset X$.

12. Kaczynski [45, Theorems 1-4]: $X$ is a subset of $E, g=1_{X}$, and $F=f \in \mathbb{C}(X, E)$ with the condition (iii). 
13. Browder [46, Theorem 9]: $X$ is a subset of a Banach space $E, F \in \mathbb{K}(X, E)$, and $g=1_{X}$ such that there is a continuous map $p: X \rightarrow[0,1]$ such that for any $x \in X$ with $x \notin F x$ we have $\operatorname{dist}(x, F x) \geq p(x)>\operatorname{dist}\left(F x, I_{X}(x)\right)$.

14. Arino et al. [47, Theorem 1]: $X$ is a subset of a metrizable locally convex Hausdorff topological vector space $E, g=1_{X}$, and $F=f \in \mathbb{C}((X, w),(E, w))$ is weakly sequentially continuous.

15. Ha [18, Theorem 4]: $X$ is a compact convex subset of a Hausdorff topological vector space, $E$ a locally convex Hausdorff topological vector space, and $F=f, g \in \mathbb{C}(X, E)$ satisfying the boundary condition (i) such that $g(X)$ and $g^{-1}(y)$ are convex for $y \in g(X)$.

16. Hadžić [31, Theorem 3]: $X$ is a subset of a normed vector space $E, F=f \in \mathbb{C}(X, E)$, and $g \in \mathbb{C}(X, X)$ is almost affine such that for each $x \in X$ with $g x \neq f x$ the line segment $[g x, f x]$ contains at least two points of $X$.

17. Ha [35, Theorem 4]: $X$ is a subset of a locally convex Hausdorff topological vector space $E, g=1_{X}$, and $F \in \mathbb{K}(X, E)$ with the condition (iii).

18. Roux and Singh [14, Theorem 5]: $X$ is a subset of $(E, \tau), g=1_{X}$, and $F=f \in$ $\mathbb{C}((X, \tau),(E, w))$ with $f x \in \bar{I}_{X}(x)$ for all $x \in X$.

19. Roux and Singh [14, Theorem 6]: $X$ is a weakly compact convex subset of $(E, \tau), g=1_{X}$, and $F=f \in \mathbb{C}((X, w),(E, \tau))$ with $f x \in \bar{I}_{X}(x)$ for all $x \in X$.

20. Lin [3, Theorem 4]: $X$ is a weakly compact convex subset of a locally convex Hausdorff topological vector space, $(E, \tau)$ locally convex, $F=f \in \mathbb{C}(X,(E, \tau))$, and $g \in \mathbb{C}(X,(E, w))$ with (ii).

21. Sehgal et al. [17, Corollary 6]: $X$ is a weakly compact convex subset of a normed vector space $E, g=1_{X}$, and $F=f \in \mathbb{C}((X, w), E)$ such that for each $x \in X$ with $x \neq f x$, the line segment $[x, f x]$ contains at least two points of $X$.

22. Ben-El-Mechaiekh [48, Theorem 4.4]: $X$ is a subset of $E, E$ is locally convex, $g=1_{X}$, and $F \in \mathbb{K}_{c}(X, X)$.

23. Ben-El-Mechaiekh and Deguire [21, Corollary 3.6], [22, Corollary 7.6]: $X$ is a subset of $E, E$ is locally convex, $g=1_{X}$, and $F \in \mathbb{A}_{c}(X, X)$.

24. Ding and Tan [9, Theorem 6$]: X$ is a weakly compact convex subset of a locally convex Hausdorff topological vector space $(E, \tau), F \in \mathbb{K}((X, w),(E, \tau))$, and $g=1_{X}$.

25. Park [29, Theorem 4]: $X$ is a subset of $E, g=1_{X}$, and $F \in \mathbb{V}(X, E)$.

26. Park [19, II, Theorem 5]: $X$ is a subset of $E, g=1_{X}$, and $F \in \mathfrak{A}_{c}^{\kappa}(X, E)$.

27. Park [19, II, Corollary 5.1]: $X$ is a subset of $E, F=f=1_{X}$, and $g \in \mathbb{C}(X, E)$ is affine such that $X \subset g(X)$ (which implies $\lambda g x+(1-\lambda) f x=x \in X \subset g(X) \subset \bar{I}_{g(X)}(g x)$ for $\lambda=0$ and $x \in X)$.

28. Ding and Tarafdar [36, Theorems $\left.3.4,3.5,3.4^{\prime}, 3.5^{\prime}\right]: F \in \mathbb{K}(X, E)$.

Finally, note that if $g=1_{x}$, then Theorem 3.2 (vi) reduces to Theorem 2.1. Therefore, in a wide sense, Theorems $2.1,3.1$, and 3.2 are equivalent.

ACKNOWLEDGEMENT. This paper is partially supported by Ministry of Education, 1996, Project No. BSRI-96-1413. 


\section{REFERENCES}

[1] FAN. K., "Extensions of two fixed point theorems of F. E. Browder," Math. Z. 112 (1969), 234-240.

[2] PROLLA, J. B., "Fixed-point theorems for set-valued mappings and existence of best approximants," Numer. Funct. Anal. and Optimiz. 5 (1982-83), 449-455.

[3] LIN, T.-C., "Some variants of a generalization of a theorem of Ky Fan," Bull. Polish Acad. Sci. Math. 37 (1989), 629-635.

[4] CARBONE, A., "A note on a theorem of Prolla," Indian J. pure appl. Math. 23 (1991), 257-260.

[5] CARBONE, A. and CONTI, G., "Multivalued maps and the existence of best approximants," J. Approx. Th. 64 (1991), 203-208.

[6] PARK, S., "On generalizations of Ky Fan's theorems on best approximations," Numer. Funct. Anal. and Optimiz. 9 (1987), 619-628.

[7] PARK, S., "On the Tychonoff-Fan type coincidence theorems," Proc. Coll. Natur. Sci. Seoul Nat. U. 14 (1989), 7-15.

[8] SESSA, S. and SINGH, S. P., "Applications of the KKM-principle to Prolla type theorems," Tamkang J. Math. 23 (1992), 279-287.

[9] DING, X. P. and TAN, K.-K., "A set-valued generalization of Fan's best approximation theorem," Can. J. Math. 44 (1992), 784-796.

[10] KAPOOR, O. P., "Two applications of an intersection theorem," J. Math. Anal. Appl. 41 (1973), 226-233.

[11] PARK, S., "Best approximation theorems for composites of upper semicontinuous maps," Bull. Austral. Math. Soc. 51 (1995), 263-272.

[12] PARK, S., "Remarks on generalization of best approximations," Honam J. Math. 16 (1994), 27-39.

[13] PARK, S., "Remarks on set-valued generalizations of best approximation theorems," Kyungpook Math. J. 35 (1995).

[14] ROUX, D. and SINGH, S. P., "On a best approximation theorem," Jñ̄anābha 19 (1989), 1-9.

[15] SEHGAL, V. M. and SINGH, S. P., "A variant of a fixed point theorem of Ky Fan," Indian J. Math. 25 (1983), 171-174.

[16] SEHGAL, V. M., SINGH, S. P., and SMITHSON, R. E., "Nearest points and some fixed point theorems for weakly compact sets," J. Math. Anal. Appl. 128 (1987), 108-111.

[17] SEHGAL, V. M., SINGH, S. P., and WHITFIELD, J. H. M., "KKM-maps and fixed point theorems," Indian J. Math. 32 (1990), 289-296.

[18] HA, C.-W, "Extensions of two fixed point theorems of Ky Fan," Math. Z. 190 (1985), 13-16.

[19] PARK, S., "Fixed point theory of multifunctions in topological vector spaces," J. Korean Math. Soc., I, 29 (1992), 191-208; II, 30 (1993), 413-431.

[20] BEN-EL-MECHAIEKH, H. and DEGUIRE, P., “ Approximation of non-convex setvalued maps," C. R. Acad. Sci. Paris Sér. I Math. 312 (1991), 379-384.

[21] BEN-EL-MECHAIEKH, H. and DEGUIRE, P., "General fixed point theorems for nonconvex set-valued maps," C. R. Acad. Sci. Paris Sér. I Math. 312 (1991), 433-438.

[22] BEN-EL-MECHAIEKH, H. and DEGUIRE, P., "Approachability and fixed points for non-convex set-valued maps," J. Math. Anal. Appl. 170 (1992), 477-500.

[23] GÓRNIEWICZ, L., "Homological methods in fixed point theory of multivalued maps," Dissert. Math. 129 (1976), 71pp.

[24] DZEDZEJ, Z., "Fixed point index theory for a class of nonacyclic multivalued maps," Dissert. Math. 253 (1985), 53pp.

[25] LASSONDE, M., "Réduction du cas multivoque an cas univoque dans les problèmes de coincidence," Fixed Point Theory and Applications (M.A. Théra and J.-B. Baillon, eds.) Longman Scientific \& Technical, Essex, 1991, pp.293-302.

[26] PARK, S., SINGH, S. P., and WATSON, B., "Some fixed point theorems for composites of acyclic maps," Proc. Amer. Math. Soc. 121 (1994), 1151-1158.

[27] BEN-EL-MECHAIEKH, H. and IDZIK, A., "A Leray-Schauder type theorem for approximable maps," Proc. Amer. Math. Soc. 122 (1994), 105-109.

[28] PARK, S., "Best approximations, inward sets, and fixed points," Progress in Approximation Theory (P. Nevai and A. Pinkus, eds.), Academic Press, 1991, pp.711-719.

[29] PARK, S., "Acyclic maps, minimax inequalities, and fixed points," Nonlinear Anal. TMA, 24 (1995), 1549-1554.

[30] FAN, K., "A generalization of Tychonoff's fixed point theorem," Math. Ann. 142 (1961), 305-310. 
[31] HADŽIĆ, O., "Some remarks on a theorem on best approximations," Rev. Anal. Num. et Th. de l'Approx. 15 (1986), 27-35.

[32] HALPERN, B., "Fixed-point theorems for set-valued maps in infinite dimensional spaces," Math. Ann. 189 (1970), 87-98.

[33] FITZPATRICK, P. M. and PETRYSHYN, W. V., "Fixed point theorems for multivalued acyclic mappings," Pacific J. Math. 54 (1974), 17-23.

[34] REICH, S., "Fixed point theorems for set-valued mappings," J. Math. Anal. Appl. 69 (1979), 353-358.

[35] HA, C.-W., "On a minimax inequality of Ky Fan," Proc. Amer. Math. Soc. 99 (1987), $680-682$.

[36] DING, X. P. and TARAFDAR, E., "Some further generalizations of Ky Fan's best approximation theorem," J. Approx. Th. 81 (1995), 406-420.

[37] REICH, S., "Fixed points in locally convex spaces," Math. Z. 125 (1972), 17-31.

[38] KNASTER, B., KURATOWSKI, C. und MAZURKIEWICZ, S., "Ein Beweis des Fixpunktsatzes für $n$-dimensionale Simplexe," Fund. Math. 14 (1929), 132-137.

[39] EILENBERG, S. and MONTGOMERY, D., "Fixed point theorems for multi-valued transformations," Amer. J. Math. 68 (1946), 214-222.

[40] HALPERN, B., "Fixed point theorems for outward maps," Univ. of California, Los Angeles, Dissertation, 1965.

[41] BROWDER, F. E., "A new generalization of the Schauder fixed point theorem," Math. Ann. 174 (1967), 285-290.

[42] HALPERN, B. and BERGMAN, G. E., "A fixed-point theorem for inward and outward maps," Trans. Amer. Math. Soc. 13 (1968), 353-358.

[43] REICH, S., "Approximate selections, best approximations, fixed points, and invariant sets," J. Math. Anal. Appl. 62 (1978), 104-113.

[44] BROWDER, F. E., "On a sharpened form of the Schauder fixed point theorem," Proc. Nat. Acad. Sci. U.S.A. 74 (1977), 4749-4751.

[45] KACZYNSKI, T., "Quelques théorèmes de points fixes dans des espaces ayant suffisamment de fonctionnells linéaires," C. R. Acad. Sci. Paris Sér I Math. 296 (1983), 873-874.

[46] BROWDER, F. E., "Coincidence theorems, minimax theorems, and variational inequalities," Contemp. Math. Amer. Math. Soc. 26 (1984), 67-80.

[47] ARINO, O., GAUTIER, S., and PENOT, J. P., "A fixed point theorem for sequentially continuous mappings with application to ordinary differential equations," Funk. Ekv. 27 (1984), 273-279.

[48] BEN-EL-MECHAIEKH, H., "The coincidence problem for compositions of set-valued maps," Bull. Austral. Math. Soc. 41 (1990), 421-434. 


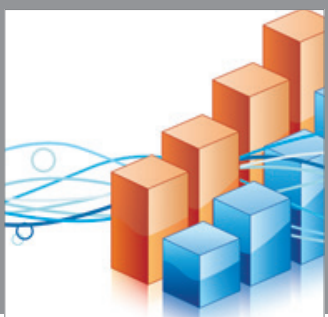

Advances in

Operations Research

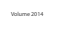

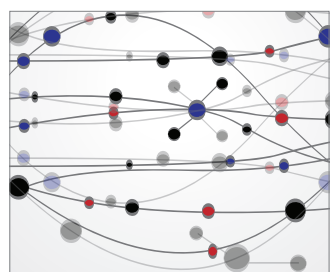

\section{The Scientific} World Journal
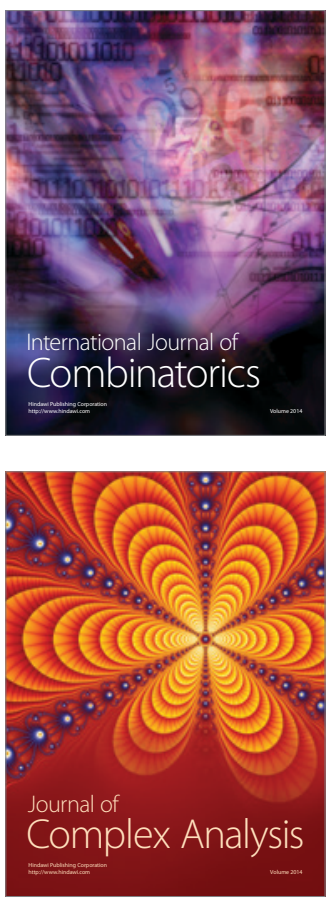

International Journal of

Mathematics and

Mathematical

Sciences
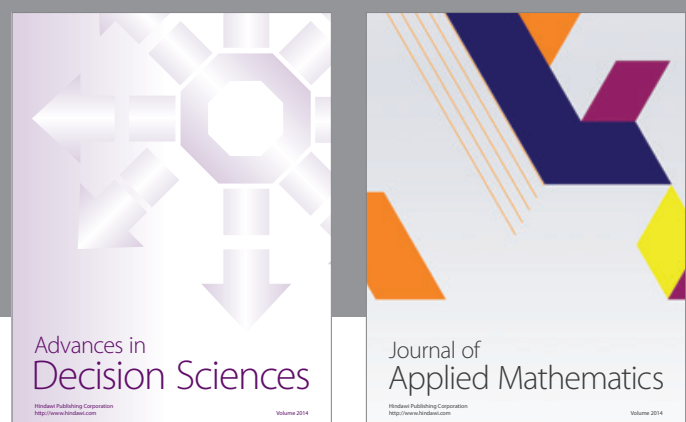

Journal of

Applied Mathematics
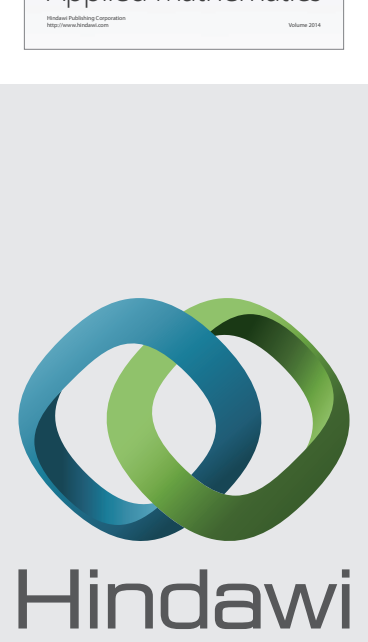

Submit your manuscripts at http://www.hindawi.com
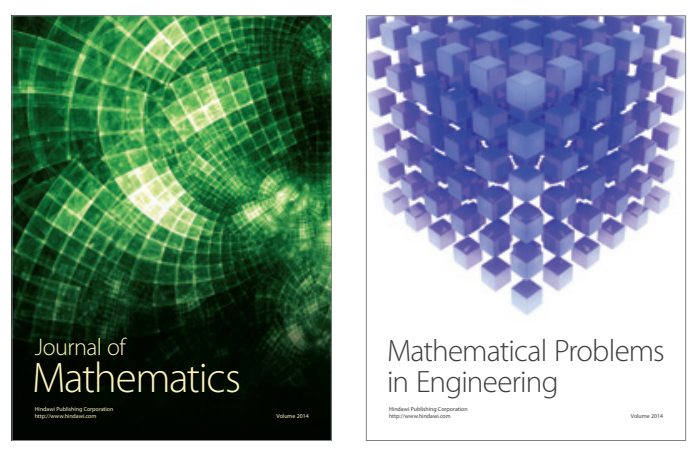

Mathematical Problems in Engineering
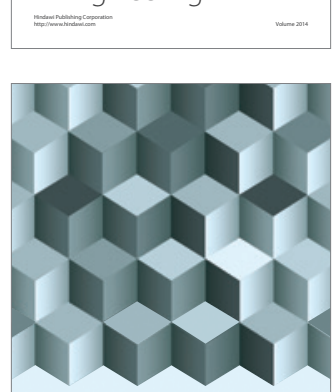

Journal of

Function Spaces
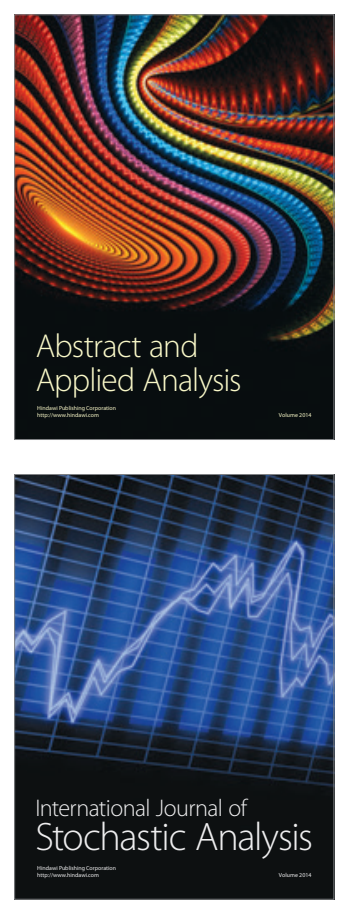

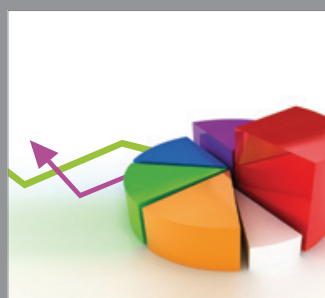

ournal of

Probability and Statistics

Promensencen
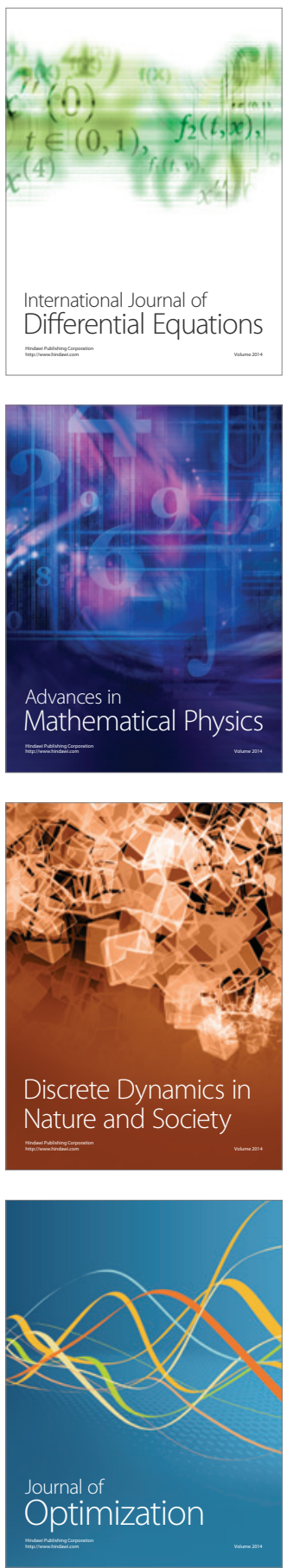